patient and staff satisfaction (Cornwell, 2012). A pressure ulcer audit (Hospice UK, 2016) on a hospice inpatient unit (IPU) highlighted the need to improve nurses' skills in the prevention, management and documentation of skin integrity.

Aim To design a comfort round tool specifically for use within the hospice IPU, implement comfort rounds and evaluate the impact on patients and nurses.

Method Nurses on the IPU were consulted regarding the overall concept, naming and design of the tool. Training was provided and following implementation, feedback sought from IPU nurses and patients regarding the impact of comfort rounds on patient care and experience.

Results Nurses' feedback: Healthcare assistants experienced job satisfaction with their involvement in continuity of patient care and evidencing care they provided. They felt areas for improvement included managing time pressures, patient choice, document design, training and team working. Patient feedback: 14 responses, all of whom were positive about the comfort rounds. They felt areas for improvement included communication about the rounds, patient choice and ensuring these discussions are communicated across the team.

Conclusion Comfort rounds have enabled our patients to feel less isolated and provided reassurances to family members. They support the consistent nursing care of our patients, including improving the involvement of the healthcare assistant workforce, optimising hydration and the prevention of pressure ulcers and falls. Initial assessment of patients admitted to the IPU needs to include discussion about the comfort rounds and creating individualised plans of care including the patient's needs and preferences relating to comfort rounds.

\section{P-172 PSYCHOSOCIAL EXPERIENCE OF WEIGHT LOSS IN CANCER CACHEXIA. WHAT CAN HEALTH CARE PROFESSIONALS LEARN?}

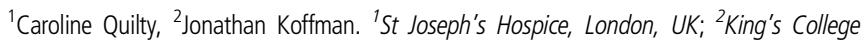
London, Cicely Saunders Institute, London, UK

\subsection{6/bmjspcare-2017-hospice.197}

Background Up to $80 \%$ of people with advanced cancer experience cachexia and of these most have accompanying anorexia, characterised by poor appetite and weight loss. Cachexia has profound psychosocial impact on patients and families including negative emotions associated with reduced food intake and involuntary weight loss, and the social consequences of these symptoms. Weight loss can be unacknowledged by healthcare professionals (HCPs), even in settings where patients are weighed regularly. Little is known about the meaning of weight loss from the patient's perspective, and what support patients expect from HCPs.

Aim(s) - To describe the experience of weight loss in people with advanced cancer and explore what psychosocial support they believe HCPs could provide

- To make recommendations for clinical practice.

Methods Individual, semi-structured interviews with hospice patients diagnosed with advanced cancer and who had expressed distress around weight loss, analysed using thematic content analysis.

Results Nine patients took part. Participants described the emotional impact of weight loss as an indicator of overall deterioration or impending death. Concerns about weight loss were perceived to be unacknowledged by HCPs, and participants would welcome more opportunities to discuss their fears. Findings were categorised into four themes:

- The meaning of weight loss to the person

- A changed relationship with food

- The experience of being weighed as 'a tick box exercise'

- 'Listen to me' - a message for healthcare professionals. Conclusions HCPs should acknowledge weight loss in patients with advanced cancer to explore the impact and meaning. Increased awareness of the impact of cachexia and communication skills training may enable these difficult conversations to take place. With appropriate emotional and social support, patients and their families can identify and address what is important to them, which may reduce distress and improve quality of life.

\section{P-173 A ROLE FOR EMOTIONAL FREEDOM TECHNIQUE IN PALLIATIVE PATIENTS? THREE CASE REPORTS}

${ }^{1,2}$ Seamus Coyle. 'Willowbrook Hospice, Prescot, UK; ${ }^{2}$ MCPCIL, University of Liverpool

10.1136/bmjspcare-2017-hospice. 198

Background Emotional Freedom Technique (EFT) is a simple and common self-help technique, which is also known as 'Tapping'. It combines elements of exposure therapy, cognitive behavioural therapy and somatic stimulation. It is widespread in the public domain; Meta-analyses show that EFT is effective for anxiety, depression and Post Traumatic Stress Disorder (PTSD). There are no studies examining the effect of EFT for palliative patients.

Case Presentation This case report presents three cases of emotional distress in palliative patients.

Case Management Each patient was treated using EFT. Case Outcome Following treatment using EFT, all of the patients' emotional distress was decreased and within a very short time. Conclusions EFT is a very simple, effective and safe technique. EFT has the potential to be a powerful tool to improve the care of palliative patients who have distressing emotions. Future research on EFT and its effect on distressing emotions in palliative populations and their families is warranted.

\section{P-174 PERSONALISED CARE PLANNING}

Tracy Bell. Rotherham Hospice, Rotherham, UK

\subsection{6/bmjspcare-2017-hospice. 199}

Background It was identified that the hospice in- patient unit records were not as robust as they could be. Despite the patients receiving an excellent level of holistic care, this was not always evidenced clearly in the documentation.

Aim and Implementation To modify existing documentation to clearly evidence the excellent care being received by our inpatients and families, whilst being patient- centred and personalised. The time frame was set for compliance to be complete by the end of January 2017. Weekly audit to ensure the new documentation is fit for purpose and to identify any areas which need further development. Following the guidelines of the NMC code for nurses and midwives specifically sections: 10 - Keep clear and accurate records relevant to your practice ... 3 - Make sure that people's physical, social and psychological needs are assessed and responded to. 